\title{
Further Interference Peaks in Mass Analysed Ion Kinetic Energy Spectra
}

\author{
Bernd Schaldach and Hans-Friedrich Grützmacher
}

Fakultät für Chemie, Universität Bielefeld, Universitätsstrasse, D-4800 Bielefeld 1, West Germany

\begin{abstract}
It is shown that interference peaks in mass analysed ion kinetic energy spectra can also occur from ions decomposing in the accelerating region of the ion source.
\end{abstract}

\section{INTRODUCTION}

Recently, Beynon and co-workers reported ${ }^{1}$ on interference peaks in mass analysed ion kinetic energy (MIKE) spectra. We have been confronted with this problem too, ${ }^{2}$ and arrived principally at the same explanations concerning the origins of certain interference peaks in MIKE spectra. ${ }^{3}$ However, in addition to the cases mentioned by Ast et al., ${ }^{1}$ there are often a lot of sharp peaks observed in MIKE spectra which do not fit the equations given by these authors and which arise from decompositions of metastable ions in the acceleration field. These interference peaks are easily distinguished from normal signals in a MIKE spectrum by their much smaller peak width (see Fig. 2). Decompositions occurring within the accelerating region of the ion source have also been considered by Lacey and Macdonald. ${ }^{4}$ Their generalized treatment of ion fragmentations within the various regions of a mass spectrometer of conventional geometry is not directly related to MIKES, however.

\section{RESULTS AND DISCUSSION}

Considering the fragmentation

$$
m_{1}^{+} \rightarrow m_{2}^{+}+m_{3}
$$

ions $\mathrm{m}_{1}{ }^{+}$continuously decompose along their flight path through the mass spectrometer. The local point of decomposition is determined by the rate constants of reaction (1) and by instrumental parameters. Those ions $\mathrm{m}_{2}{ }^{+}$which are formed within the accelerating region of the ion source will obtain a continuum of kinetic energies, given by Eqn (2)

$$
1 / 2 m_{2} v_{f}^{2}=z V_{0}\left\{\frac{m_{2}}{m_{1}} f+(1-f)\right\}
$$

where $v_{f}=$ velocity of daughter ions, $V_{0}=$ accelerating voltage and $f=$ fraction of $V_{0}$. Ions $m_{2}{ }^{+}$formed at the fraction $f$ of $V_{0}$ will pass the magnetic sector with an apparent mass $m_{f}$ given by Eqn (3)

$$
m_{f}=\frac{m_{2}^{2}}{m_{1}}\left[f+\frac{m_{1}}{m_{2}}(1-f)\right]
$$

Similarly, the electric sector voltage $E_{f}$ at which these ions pass the electric sector is given by Eqn (4) derived from Eqn (2)

$$
E_{f}=E_{0} \frac{m_{2}}{m_{1}}\left[f+\frac{m_{1}}{m_{2}}(1-f)\right]
$$

From Eqns ( 3 and 4 ) the range of apparent masses $m_{f}$ and the range of the electric sector voltages can be evaluated for reaction (1) occurring in the accelerating region of the ion source. Setting $f=0$ is equivalent to regarding ions $m_{2}{ }^{+}$generated in the ion source before acceleration, i.e. $m_{f}=m_{2}$ and $E_{f}=E_{0}$. The other extreme, $f=1$, is equivalent to observing ions $m_{2}{ }^{+}$formed in the first field free region, i.e.

$$
m_{f}=\frac{m_{2}^{2}}{m_{1}} \quad \text { and } \quad E_{f}=\frac{m_{2}}{m_{1}} \times E_{0}
$$

Thus, by preselecting $m_{f}$ values in between these extremes the fraction $f$ of $V_{0}$ [Eqn (2)] at which the observed $\mathrm{m}_{2}{ }^{+}$is formed is predetermined and can be calculated explicitly from Eqn (5)

$$
f=\frac{\frac{m_{f}}{m_{2}}-1}{\frac{m_{2}}{m_{1}}-1}
$$

Having determined $f$ the corresponding $E_{f}$ can be calculated [Eqn (4)] and the interference peak can be recorded. This is most easily done on a mass spectrometer with 'reversed geometry' by setting the magnet current to the appropriate $m_{f} / z$ value and scanning $E$ over a small region around $E_{f}$. In this way any slow fragmentation reaction can be followed step by step from the ionization chamber to the 1st field free region (FFR). ${ }^{6}$

The procedure outlined above is illustrated by the reactions shown in Scheme 1. The ranges of $m_{f}$ and $E_{f}$ values are also given in Scheme 1. Three regions of $m_{f}$ values can be distinguished in this case: (i) $149 \geqslant m_{f} \geqslant$ 145 , only ions $b$ are expected to be transmitted, (ii) $145 \geqslant m_{f} \geqslant 135$, both ions $a$ and ions $b$ can be transmitted, (iii) $134 \geqslant m_{f} \geqslant 128$, only ions $a$ will be observed. The results of the respective MIKE scans obtained with a Varian MAT 311A mass spectrometer are shown in Fig. 1 and the detailed values are given in Table 1. Thus, by tuning in the magnet to the value 


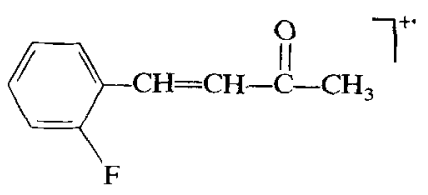

$[1]^{+\cdot}, m / z \quad 164$

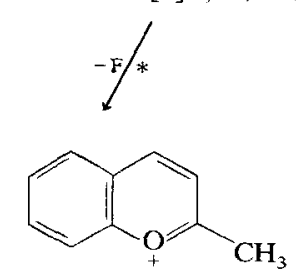

$a, m / z 145$

$m / z 145 \geqslant m_{f} / z \geqslant m / z \quad 128.2$ $E_{0} \geqslant E_{\mathrm{f}} \geqslant 0.884 V_{0}^{\mathrm{el}}$

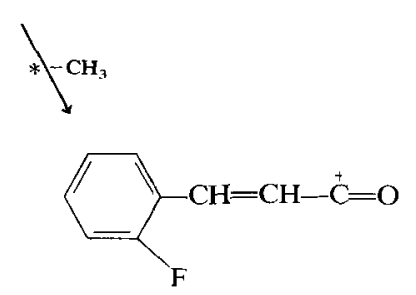

$b, m / z \quad 149$

$m / z \quad 149 \geqslant m_{\mathrm{f}} / z \geqslant m / z 135.37$ $E_{0} \geqslant E_{f} \geqslant 0.909 V_{n}^{e l}$ $m_{f} / z 147$ a sharp interference peak is expected at $E_{f}=0.9865 E_{0}$. The experiment gives an intense signal at $E_{f}=0.9866 E_{o}$ (Table 1) in excellent agreement with expectation. According to Eqn (6) (see below) this signal corresponds to ions $b$ which have been formed after the acceleration of ions $[1]^{+\cdot}$ to $f=0.1468$ (see Table 1 ). With the magnet at the value $m_{f} / z=145$, the peak for ions $b$ is shifted to $E_{f}=$ $0.9731 E_{0}$ (calc. $E_{f}=0.9731 E_{0}$ ) (see Fig. 1(a) and Table 1) and the point of formation has shifted to $f=0.2935$. In progressing to lower $m_{f} / z$ values there are now two intense sharp peaks observed [Fig. 1(bg)] at exactly the positions calculated for ions $b$ and $a$ from Eqns (4 and 5) (Table 1). In the case of $m_{f} / z 136$ and $m_{f} / z 135$ [Fig. 1(f and g)] a dramatic increase in intensity of the peak belonging to ions $b$ is observed. This is due to approaching the value $f=1$. The ions $b$ are now sampled from the 1 st FFR and the ion current is integrated over a relatively large range of lifetimes. In previous cases the sampling area and the span of lifetimes were much shorter and were related to the

Table 1. Calculated and experimental values for $E_{\mathrm{f}} / E_{0}$ for the formation of ions $a$ and $b$ (Scheme 1 ) in the accelerating region of the ion source

\begin{tabular}{|c|c|c|c|c|c|c|}
\hline \multicolumn{3}{|c|}{ Ions B } & \multicolumn{4}{|c|}{ lons a } \\
\hline$m_{t} / z$ & $\begin{array}{l}E_{f} / E_{0} \\
\text { (calc.) }\end{array}$ & $\begin{array}{l}E_{\mathrm{f}} / E_{\mathrm{o}} \\
\text { Lobs. }\end{array}$ & $t$ & $\begin{array}{l}E_{t} / E_{0} \\
\text { (calc.) }\end{array}$ & $\begin{array}{l}E_{\mathrm{f}} / E_{\mathrm{o}} \\
\text { (abs.) }\end{array}$ & $f$ \\
\hline 147 & 0.9865 & 0.9866 & 0.1468 & - & - & - \\
\hline 146 & 0.9798 & 0.9798 & 0.2201 & - & - & - \\
\hline 145 & 0.9731 & 0.9731 & 0.2935 & 1.0000 & 1.0000 & 0 \\
\hline 144 & 0.9663 & 0.9664 & 0.3668 & 0.9930 & 0.9935 & 0.0595 \\
\hline 143 & 0.9596 & 0.9596 & 0.4402 & 0.9861 & 0.9858 & 0.1191 \\
\hline 142 & 0.9529 & 0.9530 & 0.5136 & 0.9792 & 0.9792 & 0.1786 \\
\hline 141 & 0.9462 & 0.9466 & 0.5869 & 0.9723 & 0.9724 & 0.2381 \\
\hline 140 & 0.9395 & 0.9396 & 0.6603 & 0.9654 & 0.9654 & 0.2976 \\
\hline 139 & 0.9328 & 0.9328 & 0.7337 & 0.9585 & 0.9585 & 0.3572 \\
\hline 138 & 0.9261 & 0.9261 & 0.8070 & 0.9516 & 0.9516 & 0.4167 \\
\hline 137 & 0.9723 & 0.9724 & 0.8804 & 0.9447 & 0.9448 & 0.4762 \\
\hline 136 & 0.9127 & 0.9128 & 0.9538 & 0.9378 & 0.9379 & 0.5358 \\
\hline 135 & - & - & - & 0.9309 & 0.9291 & 0.5953 \\
\hline 134 & - & 一 & - & 0.9240 & 0.9239 & 0.6548 \\
\hline 133 & - & - & - & 0.9171 & 0.9168 & 0.7143 \\
\hline 132 & - & - & - & 0.9102 & 0.9099 & 0.7739 \\
\hline 131 & - & - & - & 0.9033 & 0.9032 & 0.8334 \\
\hline 130 & - & - & 一 & 0.8965 & 0.8965 & 0.8929 \\
\hline 129 & - & - & - & 0.8896 & 0.8894 & 0.9525 \\
\hline
\end{tabular}

resolving power of the magnetic sector. Thus, by opening the intermediate slit located between the magnetic and the electric sector it is possible to increase the abundances of ions formed in the accelerating region. By further lowering $m_{f} / z$, peaks due to ions $b$ are no longer observed [Fig. 1(h-l), Table 1). However, the peaks related to ions $a$ are still observed until $m_{f} / z 128$ is reached. Again a dramatic increase in intensity indicates the formation of ions $a$ in the 1 st FFR [Fig. 1(1)].

If it is necessary to determine the origin of an unknown interference peak appearing in a MIKE spectrum the difficulty arises that there are only two equations [Eqns (4 and 5)] but three unknowns $\left(m_{1}, m_{2}, f\right)$. From the combination of Eqns ( 4 and 5) it is possible to determine $m_{2}$ [Eqn (6)].

$$
m_{2}=m_{f} \times \frac{E_{0}}{E_{f}}
$$

The parent ions belonging to $m_{2}$ can be identified by applying the refocusing technique. If there is more than one parent $m_{1}$ observed, the various $m_{1}$ can be checked with the aid of Eqn (5). Negative values or values larger than 1 are not possible for $f$ and the corresponding $m_{1}$ is discarded. If there remains ambiguity one has to follow the peak by successively lowering both $m_{f} / z$ and $E_{f}$ until a steep increase in the

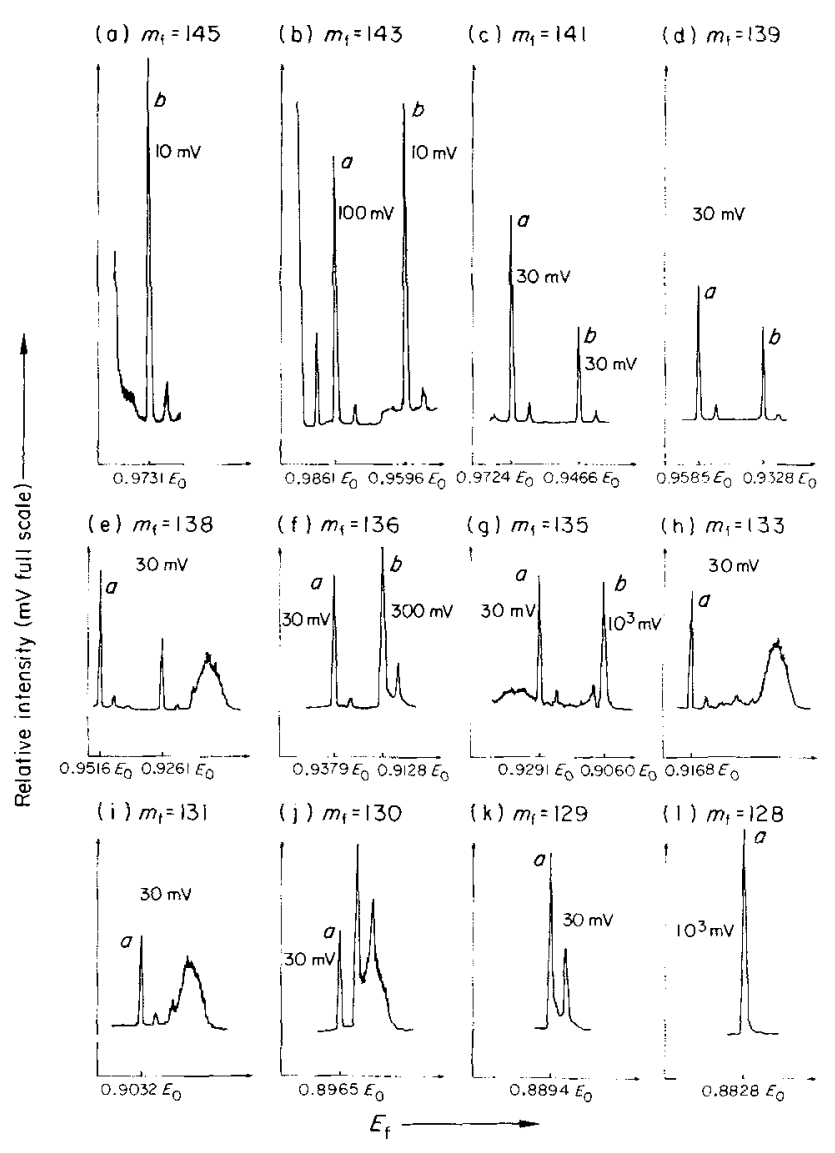

Figure 1. Interference peaks due to the formation of ions a and $b^{7}$ (Scheme 1) within the accelerating region of the ion source. 


\section{B. SCHALDACH AND H.-F. GRÜTZMACHER}

intensity of the daughter ion indicates that one has arrived at the special situation $f=1$. In this case the unknown value of $m_{1}$ can be calculated from $m_{f}$ or $E_{f}$ according to Eqns ( 3 or 4 ), respectively. The significance that interference peaks originating from decompositions within the accelerating region may have in MIKE spectra is illustrated in Fig. 2.

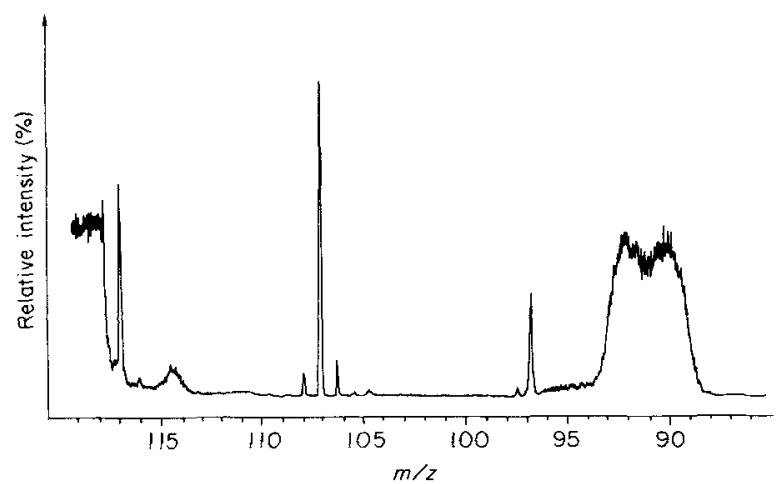

Figure 2. MIKE spectrum of ions $m / z 118$ obtained from $p$-nitrobenzalacetone.

The broad signal centred at $m / z 91$ is composed of two unresolved typical MIKE peaks. They indicate the loss of $26 \mathrm{u}$ and $28 \mathrm{u}$ in the 2nd FFR from ions $\mathrm{m} / z 118$ obtained from $p$-nitrobenzalacetone. In contrast, the sharp peaks at the apparent masses $m_{\text {app }}=$ $96.7,106.3,107.1$ and 107.9 all indicate reactions occurring in front of the magnetic analyser and which are not connected with fragmentations of jons $m / z$ 118. Application of Eqn (6) gives the various $m_{2}{ }^{+}$ ions in a straightforward way. The refocusing technique gives two parent ions $m_{1}$ for each daughter ion, $m_{2}=130$ and $m_{2}=144$, respectively (Table 2). Thus, the peak with the apparent mass 107.1 (Fig. 2) originates from two reactions occurring at different positions in the accelerating region (see Table 2 ). The ions $m_{2}=144$ also possess two parent ions. The $f$ value for $m_{1}=174$, however, is larger than 1 . To observe an intense interference peak due to this reaction, the $m_{f}$ value had to be raised to $m_{f}=144^{2} / 174=119.17$. With $m_{f}=118$, however, probably only a small part of the tail of the momentum distribution due to kinetic
Table 2. Origins of interference peaks occurring in the MIKE spectrum of ions $m / z 118$ obtained from $p$ nitrobenzalacetone

\begin{tabular}{ccccc}
\hline$E_{\mathrm{f}} / E_{0}$ & $\mathrm{~m}_{\text {aDD }}$ & $\mathrm{m}_{2}$ & $\mathrm{~m}_{1}$ & $f$ \\
0.9077 & 107.11 & 130.00 & 176 & 0.3532 \\
& & & 160 & 0.4923 \\
0.8193 & 96.68 & 144.02 & 174 & 1.0472 \\
& & & 190 & 0.7458 \\
\hline
\end{tabular}

energy release is recorded. Therefore, the predominant component of the peak with the apparent mass 96.7 (Fig. 2) belongs to the reaction of $m_{1}=190$ being accelerated to $0.7458 V_{0}$.

Besides being a nuisance in MIKE experiments the interference peaks described in this paper may give useful information about the time dependence of unimolecular reactions of ions in the gas phase. By varying $f$ and correspondingly the sampling region for metastable ions decomposing in the accelerating field of the ion source the lifetime of the metastable parent ions is also varied. Although the span of the lifetimes of reacting ions which can be investigated by this technique corresponds only to about a factor of 10 (which, however, can be enhanced by measurements at different accelerating voltages) this is of interest for certain reactions.

\section{EXPERIMENTAL}

The following conditions were used: electron energy $70 \mathrm{eV}$, emission current $2 \mathrm{~mA}$, accelerating voltage $3 \mathrm{kV}, V_{0}=510.63 \mathrm{~V}$, ion source pressure $10^{-6}-2 \times$ $10^{-6}$ Torr, ion source temperature $150^{\circ} \mathrm{C}$, direct insertion of the sample.

\section{Acknowledgement}

We thank the Fonds der Chemischen Industrie for financial support of this work.

\section{REFERENCES}

1. T. Ast, M. H. Bozorgzadeh, J. L. Wiebers, J. H. Beynon and A. G. Brenton, Org. Mass Spectrom. 14, 313 (1979).

2. B. Schaldach, Dissertation, Universität Bielefeld (1976).

3. For a differing conclusion see B. Schaldach and H.-Fr. Grützmacher, Org. Mass Spectrom. 15, (1980).

4. M. J. Lacey and C. G. Macdonald, Org. Mass Spectrom. 13, 284 (1978).

5. J. A. Hipple, R. E. Fox and E. U. Condon, Phys. Rev. 69, 347
(1946).

6. See also R. G. Cooks, J. H. Beynon, R. M. Caprioli and G. R. Lester, Metastable lons, p. 50 ff. Elsevier, Amsterdam (1973).

7. B. Schaldach and H.-Fr. Grützmacher, Int. J. Mass Spectrom. Ion Phys. 31, 257 (1979).

Received 5 October 1979; accepted 14 December 1979 (C) Heyden \& Son Ltd, 1980 